\title{
Structural features of algebraic quantum notations
}

\author{
Elizabeth Gire \\ Department of Physics, Oregon State University, Corvallis, Oregon 97330, USA \\ Edward Price \\ Department of Physics, California State University, San Marcos, California 92096, USA
}

(Received 29 September 2014; published 23 September 2015)

\begin{abstract}
[This paper is part of the Focused Collection on Upper Division Physics Courses.] The formalism of quantum mechanics includes a rich collection of representations for describing quantum systems, including functions, graphs, matrices, histograms of probabilities, and Dirac notation. The varied features of these representations affect how computations are performed. For example, identifying probabilities of measurement outcomes for a state described in Dirac notation may involve identifying expansion coefficients by inspection, but if the state is described as a function, identifying those expansion coefficients often involves performing integrals. In this study, we focus on three notational systems: Dirac notation, algebraic wavefunction notation, and matrix notation. These quantum notations must include information about basis states and their associated complex probability amplitudes. In this theory paper, we identify four structural features of quantum notations, which we term individuation, degree of externalization, compactness, and symbolic support for computational rules. We illustrate how student reasoning interacts with these structural features with episodes from interviews with advanced undergraduate physics majors reasoning about a superposition state of an infinite square well system. We find evidence of the students coordinating different notations through the use of Dirac notation, using an expression in Dirac notation to guide their work in another notation. These uses are supported by the high degree of individuation, compactness, and symbolic support for computation and the moderate degree of externalization provided by Dirac notation.
\end{abstract}

DOI: 10.1103/PhysRevSTPER.11.020109

PACS numbers: 01.40.Fk, 01.40.Ha

\section{INTRODUCTION}

External representations of physical phenomena are an important part of doing, communicating, and learning physics [1]. Expert physicists fluently use diagrams, graphs, and equations to communicate information about physical situations and as computational tools for solving problems. Nevertheless, students often have difficulty creating and using these disciplinary representations [2-7].

Quantum mechanics is particularly rich with representations. Multiple notational systems are commonly used for representing quantum systems, including, algebraic wave functions, matrices, and Dirac notation. Research on students' understandings and competencies in quantum mechanics has largely focused on students' conceptual understandings and performance in making calculations [8-13]. Some work has specifically investigated how students interpret graphs of wave functions [13-15]. Recently, as a "spins first" instructional approach has been gaining popularity, research tasks presented in Dirac notation have become more common. However, these

Published by the American Physical Society under the terms of the Creative Commons Attribution 3.0 License. Further distribution of this work must maintain attribution to the author(s) and the published article's title, journal citation, and DOI. studies have also generally aimed at identifying students' conceptual understandings or identifying common student difficulties with making calculations [16,17].

A theoretical framework for understanding student performance with quantum mechanical representations would be useful. Ultimately, such a theory would bridge these studies and more general frameworks for student learning such as misconceptions, $p$-prims, and noviceexpert frameworks [18-21]. A first step is understanding the representations themselves and their affordances for supporting computation. To this end, we have investigated several representations in quantum mechanics in terms of their features, how they support computation, and the possible implications for student learning. This paper specifically examines notational systems for quantum mechanical states with a goal of characterizing the representations. The resulting theoretical analysis is illustrated with student use of quantum mechanics notations as documented in an exploratory study of students' fluency with different notations for representing quantum states and how the features of these notational systems influence student reasoning.

\section{A. Theoretical perspectives}

We share Kirsh's [22] view that the generation and use of external representations is an interactive process: 
"By 'interactive' I mean a back and forth process: a person alters the outside world, the changed world alters the person, and the dynamic continues" (see Ref. [22], p. 171). We use ideas from distributed cognition to think about the role of external representations in these interactions. From this perspective, external representations can be thought of as artifacts having both material (physical) and ideal (conceptual) aspects [23]. Hutchins $[24,25]$ describes how material features can support computation; for example, a slide rule uses logarithmic scales printed on sliding pieces to transform multiplication into addition performed by physically combining lengths. In previous work [26], we focused on how the material features of the vector arrows representation of electric fields supports computation through the physical and geometric properties of the representation as compared to an algebraic representation of electric field. When we add vector arrows "tip to tail," the representation does some of the calculation for us. In this paper, we examine the structural features of three algebraic notational systems. All three notations share similar material features: they all employ alphanumeric symbols and are manipulated using algebraic rules. For these notational systems, the conceptual features of the notations play a more central role in our analysis.

For external representations that are primarily symbolic, Hutchins suggests that the material features are less important, compared to examples like the slide rule where computation is built into the physical object. Hutchins claims that "for symbols that have arbitrary relations to the things they denote, the material structure provides only the perceptual identity of the physical form as distinct from other physical forms" [25] and defines individuation as "the notion that some material element is different from or distinguishable from other elements" [25]. According to Hutchins, the level of computation encoded into material properties is lower for language than a slide rule. (Hutchins, however, was considering spoken and written language, not mathematical symbols.) In this view, when vectors are added algebraically (componentwise), the representation "merely" holds and displays the symbols. Kirsh extends this view by noting several ways that external representations can aid cognition, such as by acting as sharable objects of thought, being rearrangeable, having physical persistence, and allowing reformulation [22].

In our view, these functions of external representations arise from their spatial and temporal properties. Temporal persistence allows external representations to store information (i.e., "remember") and to be shared across individuals. The construction of external representations is sequential, facilitating the process of orienting the external representation to other representations (like mental representations or text and (or) verbal descriptions). The spatial extent of a representation allows for the representation to be rearranged or for elements to be coordinated by the physical positioning. A thorough exploration of these issues is beyond the scope of this paper, but we suggest that Kirsh's features can be understood as arising from spatial and temporal properties.

In this paper, we examine structural features of notational systems for quantum mechanics and discuss how these features may be linked to the ways students generate and use external representations of quantum states. The structural features are both material and conceptual in nature. They include the shapes and spatial distribution of symbols (material features) and also the physical meanings of the symbols (conceptual). We focus on three notational systems-Dirac notation, algebraic wavefunction notation (or simply, wave-function notation), and matrix notation - and their use in representing a quantum state and computing an expectation value. We illustrate our analysis with episodes from problem-solving interviews with advanced undergraduate students.

Our exploratory inquiry has been guided by two questions:

(1) How can we characterize different representations of quantum systems based on their material and conceptual features?

(2) How do the features of these representations support or hinder students' computation?

To begin addressing these questions, we conducted interviews with advanced undergraduate physics majors. The interview tasks were designed to have students use multiple notations to represent a superposition state and perform computations on that state. While reviewing video of the interviews, we attended to differences in student performance with the different notations and instances where students coordinated multiple notations. In an attempt to explain our observations of student performance, we identified some structural features that differ across the notational systems.

We begin this paper by describing the interview task given to students. Next, we introduce some of the student reasoning that we aim to explain with a framework of structural features. We then describe a scheme for characterizing the structural aspects of different notational systems in quantum mechanics and apply this scheme to Dirac, wave-function, and matrix notations. We illustrate the consequences of these differences with examples from student interviews.

\section{INTERVIEWS}

We conducted problem-solving interviews with $n=8$ advanced undergraduate physics majors at Oregon State University $[27,28]$. The interviews were semistructured in nature and students were asked to think aloud as they were performing the interview tasks [29]. This paper focuses on how students represented a superposition state and how they computed the expectation value of energy. Student 
spent about 30 min discussing the quantum mechanics tasks. The interviews were videotaped and segments of interest were transcribed for analysis.

Students at Oregon State University take three threeweek-long "paradigm" courses on quantum mechanics in the winter quarter of the junior year and a ten-week-long "capstone" quantum mechanics course in the winter quarter of the senior year. The interview subjects included six juniors and two seniors. The interviews took place 4 weeks after the end of the winter quarter. All of the examples of student reasoning included in this paper are taken from interviews with juniors.

The three quantum paradigms courses include Spin and Quantum Measurement, Waves in One Dimension, and Central Forces. A modern physics course is a prerequisite for these courses. Spin and Quantum Measurement introduces the postulates of quantum mechanics by examining 2 and 3 state systems through computer simulations of Stern-Gerlach experiments. The Waves in One Dimension and Central Forces courses include both classical and quantum mechanics topics. The quantum content of Waves in One Dimension includes discussion of solving the Schrödinger equation in one dimension, particularly the infinite and finite square well. The quantum content of the Central Forces course focuses on solutions of the 3D Schrodinger equation for the hydrogen atom. The primary quantum textbooks for the quantum paradigms and capstone courses are Quantum Mechanics: A Paradigms Approach by McIntyre, Manogue and Tate and Introduction to Quantum Mechanics by Griffiths [30].

\section{A. Interview tasks}

Students were first asked, "So I want you to imagine that you have a particle in an infinite square well. And the well has width $L$ and one of the edges of the well is at $x=0$. Imagine that you know that probability of measuring the energy of the particle to be the ground state energy is one quarter, and the probability of measuring the first excited state energy is three quarters. How would you represent the state of the particle?" After the students exhausted their own suggestions for representations, the interviewer suggested additional representations, including a graph of a wave function, an algebraic expression of the wave unction, Dirac notation, and matrix notation to ensure that all of the representations of interest to us were discussed. One student also suggested using a histogram of energies to represent the quantum state.

The students were then asked to calculate the expectation value of the energy for this state. The students were allowed to initially proceed with whatever representation they wanted, but then the interviewer asked the students to perform the calculation using the various representations discussed earlier in the interview.

\section{B. Analysis}

Videos of the interviews were viewed and discussed by both of the authors in order to increase the reliability of our interpretations of the interviews. Although our goal in this study is not to measure trends in student reasoning but to identify an explanation of student performance with different quantum notations, we also wanted to be sure that we attended to particular aspects of student performance that might be representative of larger populations, rather than instances of highly idiosyncratic behaviors. To this end, we initially identified commonalities among the students in the types and ordering of representations suggested by the student and the interviewer, as well as the students' performance with and attitudes about each notational system. Our experiences in teaching quantum mechanics also aided us in identifying student reasoning and behavior that may be common to students at this level. We then identified specific episodes where the features of the notation appeared to influence the students' work as well as instances where students seemed to coordinate multiple notations. Narratives of these episodes were created and discussed until both authors reached an agreement on how to interpret the role of the external representation in students' reasoning.

In parallel, we analyzed the notations themselves from the perspective of distributed cognition. Starting with a comparison of how the notations represent states in quantum mechanics, we identified a set of features to characterize the different notations. We then reexamined the interviews to determine if these features of the notations were consistent with students' work.

We share Kirsh's view that the generation and use of external representations is an interaction. In our analysis, we sought to explore how the interactions between students and their external representations may be different for various notational systems, depending on the nature and features of the notation. The examples of student work presented in this paper were selected as particularly illustrative examples of how the notational system may influence student reasoning about quantum systems or for being representative of general trends of student reasoning seen in the interviews.

\section{Limitations}

Because of the small number of interviews conducted and the unique nature of the Oregon State University Paradigms in Physics program, we urge caution in generalizing the patterns of behavior and reasoning reported here to larger populations. Paradigms in Physics emphasizes the use and coordination of different notations and strongly encourages students to share explanations of their thinking (through small group and whole class discussions). Thus, we expected these students to be comfortable articulating their thinking in an interview setting and to be familiar with several different representations of quantum systems, 
including wave-function graphs, algebraic wave functions, Dirac notation, and matrix notation. Additionally, the paradigms program takes a spins first approach in teaching quantum mechanics. These students' understandings and use of representations (particularly Dirac and algebraic wave-function notations) may be different from those of students who experience a more traditional "wavefunctions-first" approach. Our intent here is not to make claims about patterns of student reasoning that may be generalized to a broader population, but is instead to illustrate how the characteristics of the notational systems interact with student reasoning while representing a superposition state and an expectation value computation.

\section{OVERVIEW OF INTERVIEW RESULTS}

We begin with some general observations about students' responses to the interview prompt. The student behaviors we identified served to guide our investigation of the structural aspects of the different notational systems.

During the interviews, students were asked to use as many different representations as they could, and prompted to use ones they did not employ on their own. We observed many instances of students moving between different representations, sometimes spontaneously, sometimes at the interviewer's prompting. Students' spontaneous coordination of different representations frequently had a sense-making function. We saw several students using an expression in one notation as a template to create the corresponding expression in another notation. In most of these cases, a linear combination of kets in Dirac notation served as a template for a superposition state. (Section IV describes why Dirac notation lends itself to this template function.) Figure 1 shows how Carlton used Dirac notation as a template for an expression in matrix notation. Carlton was prompted to represent the state in matrix notation. Starting on a clean whiteboard, he first wrote the state in Dirac notation (which he had determined earlier in the interview), then wrote a parallel expression below with column vectors in place of kets (see Fig. 1). Carlton was not sure what to put in the columns, and first put generic constants. Then, when considering the Dirac expression, he mentioned the orthornomality of the basis states and suggested that the columns should be $1000 \ldots$ and $0100 \ldots$. As another example of using Dirac notation as a template, Seth was asked to show how he could calculate the probability of finding the state in the first excited state in matrix notation. He started by writing a square bracket, presumably the beginning of a row vector. Then he erased it, saying, "I guess we'll write in bra-ket to compare it first," and wrote an expression in Dirac notation. Without pausing, he went on to write the calculation in matrix notation. When asked to compute the expectation value, he proceeded in the same way. Figure 3 shows the relevant part of his whiteboard.

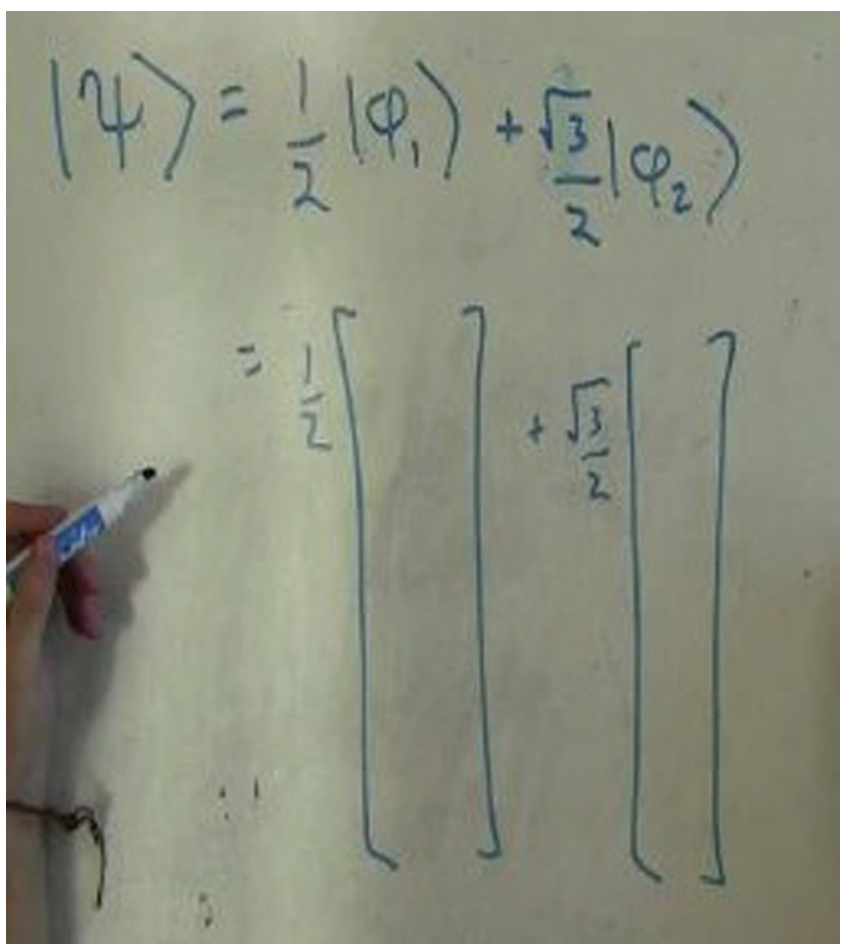

FIG. 1. Prompted to represent the state in matrix notation, Carlton begins by writing an expression in Dirac notation, then writing a parallel expression with column vectors in place of kets.

Most students had difficulty remembering the specific formula for the energy eigenstates of the infinite square well in wave-function notation. All students used the position basis when working with wave functions (as opposed to momentum), but none of them discussed this choice explicitly. There was some evidence that students believe the kets are wave functions, or at least are equivalent to wave functions. For example, Diego set a ket equal to a wave function, as illustrated in Fig. 2. Initially, he wrote a superposition state in Dirac notation in the upper left of his whiteboard. He then wrote a general form for the energy eigenstate in wave-function notation in the lower right. Then, just below this, he wrote expressions for the ground state and first excited state wave functions, setting them equal to the kets $|\mathrm{GS}\rangle$ and $|1 \mathrm{Ex}\rangle$, respectively. Finally, going back to the upper left of the board, he wrote the linear combination in wave-function notation.

Most students needed prompting to use matrix notation, and many were uncomfortable with this notation. We observed students being uncertain about what basis to work in (energy or position), as well as the form and syntax for matrix operations. We observed students mixing up column and row vectors and having difficulty putting vectors and operators in the correct order for calculations of expectation value. The students interviewed generally expressed a preference for Dirac notation and a preference not to use matrix notation if they could help it. 

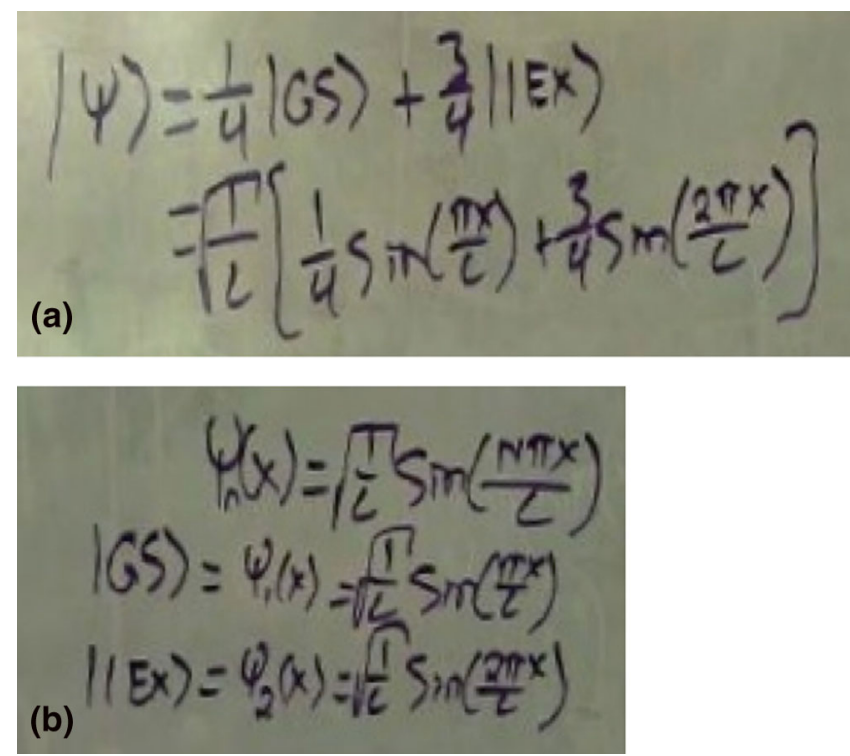

FIG. 2. Diego writes expressions with kets equal to wave functions in (a) the upper left of his whiteboard and (b) the lower right of his whiteboard. The first line in (a) was written first. Then the equations in (b) were written (in order from top to bottom). Finally, he wrote the second line in (a).

To summarize, we observed several interesting aspects of student reasoning in the interviews as follows:

- using Dirac notation as a template for writing down a superposition state in a different notation or for setting up an expectation value calculation in a different notation,

- errors in the order of the integrand for an expectation value calculation in wave-function notation,

- errors in the order of matrices for an expectation value calculation in matrix notation,

- generally making fewer errors and more progress with Dirac notation.
We sought to explain these observations during our exploration of the structural aspects of the different notational systems. The framework we developed is described in the following section.

\section{STRUCTURAL ASPECTS OF QUANTUM REPRESENTATIONS}

Two physical aspects are encoded in external representations of quantum states: the states that result from measurements (basis elements of a Hilbert space) and their associated probabilities (complex probability amplitudes). The various notational systems encode these aspects differently, and we analyze this in terms of the notational system's structural features. Through this analysis, we identify four important characteristics that we refer to as individuation, externalization, compactness, and symbolic support for computation. These are manifest in varying ways and degrees in Dirac, wave-function, and matrix notations. Table I summarizes these findings. This section describes this analysis and discusses some of the ways these characteristics support or inhibit student reasoning.

\section{A. Individuation}

Individuation is the degree to which important features are represented as separate and elemental (i.e., as an indivisible item) [31]. In Dirac notation, quantum basis states are written as kets that have a high degree of individuation. For example, when kets are added in a superposition, common terms may be factored out of the coefficients, but the individual kets remain in the sum. Quantum states written in matrix form are also individuated, with each entry in the quantum state column matrix corresponding to one of the quantum state basis vectors. Although quantities can be factored out or multiplied through, according to the rules of matrix algebra, one

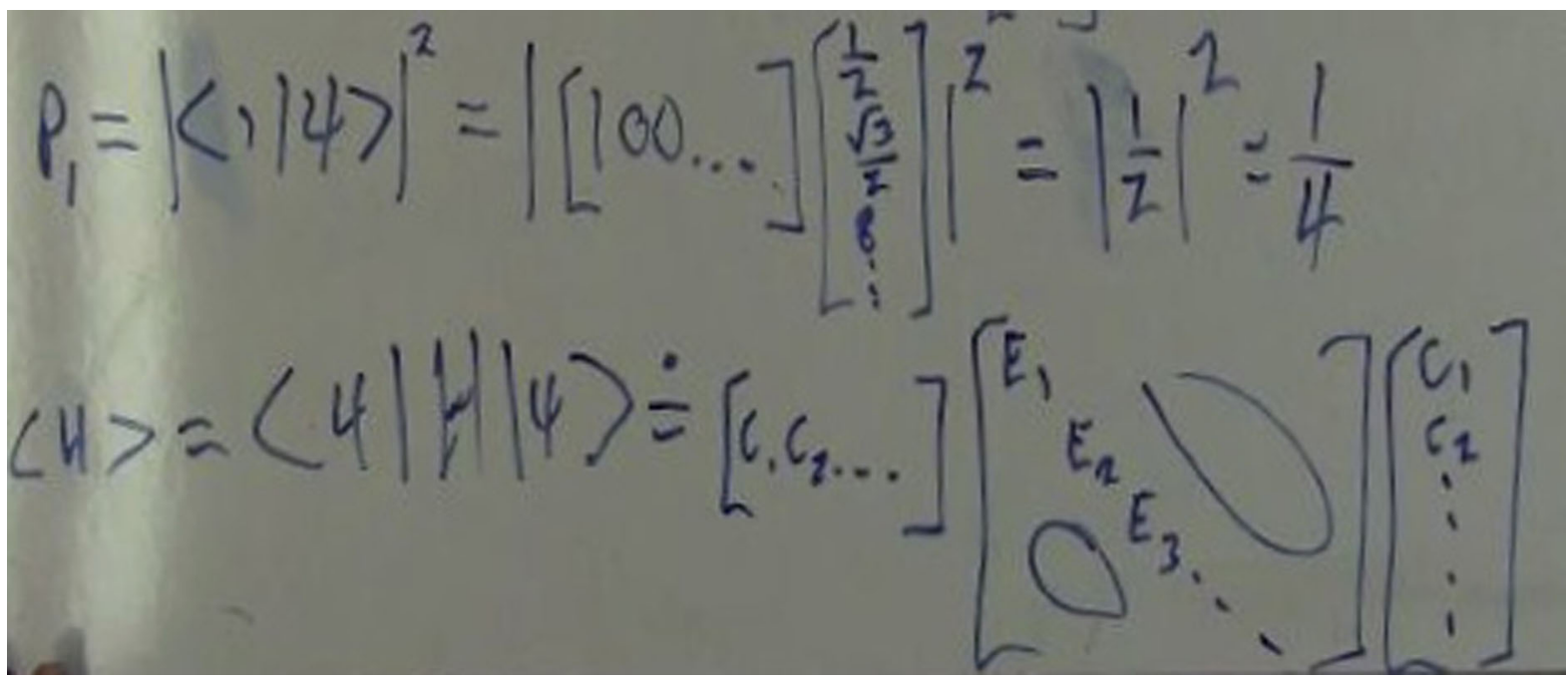

FIG. 3. Seth first uses Dirac notation as a template for an expression in matrix notation. 
TABLE I. Summary of the characteristics of individuation, externalization, compactness, and symbolic support for computation as they are manifest in notational systems for quantum mechanics.

\begin{tabular}{|c|c|c|c|c|}
\hline Notation & Individuation & Externalization & Compactness & Symbolic support \\
\hline Dirac & $\begin{array}{l}\text { High. Kets provide an } \\
\text { elemental } \\
\text { representation of } \\
\text { basis states }\end{array}$ & $\begin{array}{l}\text { Moderately low. Label } \\
\text { for quantum state is } \\
\text { ambiguous }\end{array}$ & $\begin{array}{l}\text { High for individual kets (only a } \\
\text { few simple symbols). Low } \\
\text { for superposition states with } \\
\text { many components; kets } \\
\text { cannot be combined }\end{array}$ & $\begin{array}{l}\text { High. The asymmetry of the } \\
\text { bra-ket notation makes an } \\
\text { inner or outer product easily } \\
\text { distinguishable }\end{array}$ \\
\hline Wave function & $\begin{array}{l}\text { Low. Coefficients, } \\
\text { normalization } \\
\text { constants, and basis } \\
\text { states may be } \\
\text { combined } \\
\text { algebraically }\end{array}$ & $\begin{array}{l}\text { High. Aspects such as } \\
\text { functional form, } \\
\text { periodicity, etc., are } \\
\text { explicit }\end{array}$ & $\begin{array}{l}\text { Low for individual eigenstates, } \\
\text { but may be high for infinite } \\
\text { superpositions. Basis states } \\
\text { require many symbols, but } \\
\text { superposition states may be } \\
\text { expressed compactly, e.g., } \\
\text { via Fourier synthesis }\end{array}$ & $\begin{array}{l}\text { Low. The symbols themselves } \\
\text { provide little clue on how to } \\
\text { perform operations }\end{array}$ \\
\hline Matrix & $\begin{array}{l}\text { High. Individual } \\
\text { entries in a vector } \\
\text { correspond to a } \\
\text { basis state }\end{array}$ & $\begin{array}{l}\text { Low. Nothing indicates } \\
\text { the basis elements } \\
\text { beyond the implicit } \\
\text { designation of a space } \\
\text { for an entry in the } \\
\text { vector }\end{array}$ & $\begin{array}{l}\text { High for low dimension spaces } \\
\text { such as spin- } 1 / 2 \text { systems, but } \\
\text { low for infinite-dimensional } \\
\text { Hilbert spaces }\end{array}$ & $\begin{array}{l}\text { Moderately low. Differently } \\
\text { shaped row and column } \\
\text { vectors facilitate } \\
\text { remembering to include one } \\
\text { of each in inner and outer } \\
\text { products, but provide no clue } \\
\text { as to the ordering }\end{array}$ \\
\hline
\end{tabular}

cannot merge the entries in a column vector. In contrast, quantum states represented in algebraic wave-function notation are less individuated. A linear combination of eigenstates may be compressed into an equivalent expression where the original basis states are not visible on inspection. For example, sine functions representing basis states can be combined through trigonometric identities.

Individuation affects the extent to which probability amplitudes and quantum states are distinct, and provides insight into the interviewees' work with these concepts. When writing down quantum states, nearly all the interview subjects included the expansion coefficients and basis elements of the Hilbert space in a way we refer to above as a linear combination template. These students identified each expansion coefficient with a single-letter symbol, e.g., $a|1\rangle+b|2\rangle$ versus $a e^{i \alpha}|1\rangle+b e^{i \beta}|2\rangle$. Students discussed using this template to "read off" probabilities of measurement outcomes, particularly in Dirac notation, an ability facilitated by the high degree of individuation in that notation. Using a letter symbol for the expansion coefficient for each term aids students in identifying the expansion coefficient as a separate entity from the basis elements (i.e., the two physical aspects are distinguished). In wave-function notation, however, expansion coefficients may be algebraically combined with normalization constants, thereby obscuring their distinct meanings.

While Dirac notation supports distinguishing expansion coefficients from basis elements, using a single letter to represent the expansion coefficient does not support students in attending to the nature of these expansion coefficients (or probability amplitudes in the context of quantum mechanics) as complex numbers with real and imaginary parts. In quantum mechanics, the overall complex phase of the quantum state has no physical meaning, but the relative phase between terms in the expansion is important for distinguishing different quantum states. In the interviews, only one student acknowledged the complex nature of the probability amplitudes by explicitly assuming that the probability amplitudes were real. It is worth noting that the students did not need to explicate the relative phase during the interview - the problems posed in the interview did not require the complex phase to be determined. However, the relative phase is generally an important property of superposition states.

The students were prompted to identify the quantum state based on probabilities of two energy measurements. Students who might have attended to the complex nature of the probability amplitudes could have left the imaginary phases of each term as unknowns, or followed convention by factoring out an overall phase, leaving the relative phase on the second term as an unknown. No student did this. Instead, many of the students identified the probability amplitudes by inspection, describing the probability amplitudes as the "square root of the probabilities." Some students were able to explain how to compute the probability amplitudes by setting the probabilities equal to the norm squared of the projection of the eigenstate on the superposition state. However, these students seemed to ignore the complex nature of the norm squared for the purpose of identifying the probability amplitude. While we do not suggest that the use of a single symbol for the coefficient caused this omission, it does not require students to grapple with the complex nature as other representations might (for instance, phasors). 


\section{B. Degree of externalization}

We use externalization to describe the degree to which elements and features are externalized with markings included in the representation. Wave-function notation externalizes many of the details of the quantum system. Both the basis states and the probability amplitudes, as well as the detailed mathematical behavior of the basis states (such as sinusoidal behavior and the wave number of the eigenfunctions for the infinite square well), are explicitly denoted. The externalization of these details allows wavefunction notation to be useful for computations involving regions of space, such as calculating the probability of finding a particle to be in the right half of an infinite well. Matrix and Dirac notations do not support those computations.

The externalization in Dirac notation is somewhat limited. Kets do not make the mathematical details of the quantum system explicit; a particular quantum state has only a brief ket label. In the interviews, students frequently used labels 1 and 2 for the ground state and first excited state of the infinite square well. These brief labels do not reveal details about the behavior of the quantum state. For example, the ground state of the infinite square well and the ground state of the quantum harmonic oscillator might both be represented by $|1\rangle$. The differences between the quantum states for these two different situations are much more readily detected when the states are represented as wave functions.

Matrices require spatially distributed entries for each basis element but do not require any marking to express the basis elements - each is implicitly represented by a space designated for an entry in the column (or row) vector. This lack of explicit denotation of the basis can lead to confusion in identifying the basis when students are interpreting a matrix quantum state or when trying to write down a quantum state as a matrix. Perhaps in an effort to manage this ambiguity in the basis, we observed students translating a superposition state from Dirac notation to a matrix with an energy basis by writing a separate column vector for each ket and adding them together into a single column, rather than treating each entry in the resultant column as corresponding to a ket. We also saw some students struggle to decide if the basis of the matrix was the energy eigenstates or the position basis.

A couple of students tried to write down the eigenstate wave functions of the infinite square well before doing any computation in that notation. One student wrote down the eigenstate wave functions immediately after the interviewer mentioned the infinite square well as the problem situation. By externalizing the details of a quantum state, working memory is freed for other tasks. However, a representation with less externalization may be useful in cases when the student does not remember the details of a particular quantum state. For example, we saw students abandon working with wave functions in favor of Dirac notation when they did not recall that the eigenstates of the infinite square well are sine functions or when they did not remember the wave number for each eigenstate.

\section{Compactness}

We use compactness as a measure of how much space and writing is needed. More markings generally lead to a less compact representation, and some quantum notations require more extensive markings than other notations. In thinking about the compactness of a notation, it is helpful to consider the cases of representing basis elements and superposition states separately.

Individual kets are compact. They require only a few markings to represent basis elements: a vertical line, an angle bracket delimiter, and a label. In general, wavefunction basis elements (e.g., the normalized sinusoidal functions for the infinite square well) are less compact than kets, requiring strings of alphanumeric symbols. In matrix notation, the basis states themselves require no markings, but are merely indicated by the existence of an element in a matrix.

However, for superposition states, a ket is required for each basis element with a nonzero expansion coefficient, which can be inconveniently large for infinite-dimensional Hilbert spaces. Wave functions may be algebraically simplified to represent a superposition of an infinite number of basis elements with just a few alphanumeric symbols. Matrices occupy little space for finitedimensional Hilbert spaces (e.g., spin-1/2 systems), but may be inconveniently large for infinite-dimensional Hilbert spaces (although they may be truncated). In the case of matrix notation, even though the basis states are not indicated with any marking (but merely by the existence of an element), the spatial extent of the matrix reduces the compactness of a quantum state vector.

The compactness of a representation influences how easily it can be manipulated for computation. First, more compact notations take less time to write down, making them preferable for computations that require many manipulations and rewriting of the state. Many students expressed a resistance to using matrix notation because it required a lot of writing. Second, compactness facilitates chunking pieces together into conceptual units. For example, one may think about writing down a ket $|\psi\rangle$ rather than thinking about writing a vertical line, a $\psi$, and a right angle bracket. Compact notations with a few pieces (kets) are easier to chunk than notations requiring many pieces, as wave function and matrix often do. In the interviews, many students had difficulty remembering the specific form for the infinite square well energy eigenstates in wave-function notation. As discussed in Sec. III, Seth used Dirac notation as a template for creating expressions in matrix notation (see Fig. 3). One explanation of this behavior is that it is easy for Seth to create the correct expression in the more compact Dirac notation; once the form of the expression is 
captured, he can concentrate on writing down the details of the less compact matrix expression.

\section{Symbolic support for computation}

The symbols used in each notational system support computation differently by virtue of their properties, such as physical shape. These properties are different from conventional or conceptual rules for operations, but can support those rules to varying degrees. As an example, matrix manipulations are governed by a set of rules that rely on the spatial arrangement of matrices. A row can be multiplied by a column with the same number of elements. When a row is multiplied by a column, the result is a scalar. When a column is multiplied by a row, the result is a square matrix (i.e., an operator). However, matrix notation only weakly includes visual cues for the differences in these sequences. The different shapes of row and column vectors make it easy to recall that one of each should be included, but provide no clue as to the ordering. Instead, one must simply remember that a column multiplying a row gives a matrix and vice versa. In contrast, the asymmetry of Dirac notation kets, with a straight vertical line on one side and an angle bracket on the other, signals to students the correct ordering of elements to produce either a scalar or an operator. This strong visual cue reduces the reliance on remembering a rule and allows students to quickly and reliably use Dirac notation. Wave-function notation, in contrast to Dirac and matrix notations, offers little symbolic support for the ordering of elements, except that differential operators should be followed by a function.

In the interviews, students rarely made errors with the order of elements in Dirac notation; such errors were occasional in wave-function notation and common in matrix notation. Because symbolic support plays a role in computation, it is best illustrated by looking at an episode of a student performing a computation rather than snapshots of the students' work. For this reason, we next present an extended narrative of an episode containing two examples of the symbolic structures of Dirac notation providing computational support.

When the student (Nelson) is asked to calculate the expectation value of the energy, he immediately wrote down the Dirac bracket $\langle\psi|\hat{H}| \psi\rangle$ [Fig. 4(a), upper left]. Then he described the expectation value as a weighted average. When writing the equation for performing the weighted average, he said he wanted the probabilities times the eigenvalues of the Hamiltonian, but described those eigenvalues as the "sine terms" and used his symbol "rho" for the eigenfunctions in his equation [Fig. 4(a), lower left].

The interviewer then asked the student to discuss how the calculation would proceed in Dirac notation. Nelson wrote down a Dirac bracket for the computation using the superposition states written as a sum of kets, but he
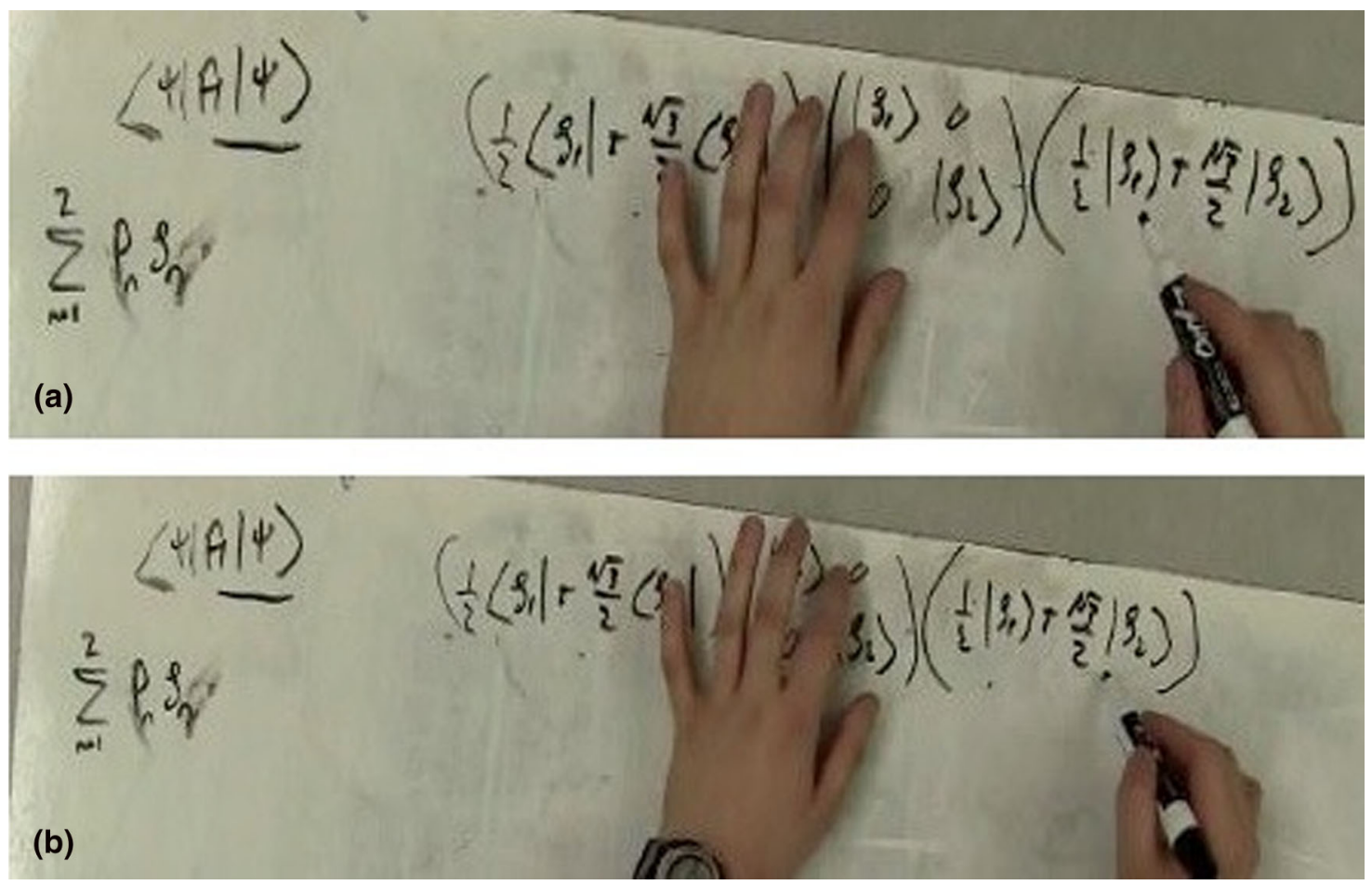

FIG. 4. (a) Calculation of energy expectation value in Dirac notation, but the student has written the Hamiltonian as a matrix and is attempting to perform matrix multiplication. In this picture, the student is multiplying to the $H_{11}$ and $\psi_{11}$ elements. (b) The student is continuing the "matrix multiplication" by multiplying the $H_{12}$ and $\psi_{12}$ elements. 
was unsure of how to represent the Hamiltonian for doing this computation. He attempted to (incorrectly) write the Hamiltonian as a diagonal matrix. He stated that the elements along the diagonal should be the energy eigenvalues, but when writing the matrix, he put the eigenstate kets along the diagonal [Fig. 4(a)]. "Well, it's a, $\mathrm{H}$ is a diagonal matrix with its eigenvalues down the diagonal. And apply this (Hamiltonian operator) on to (the superposition state)." He then performed a "matrix multiplication" between the Hamiltonian matrix and the superposition state, treating each term in the superposition as an element in a row matrix and pointing to the elements to be multiplied together as shown in Figs. 4(a) and 4(b). "So, matrix multiplication, this $\left(H_{11}\right.$ element) times this $\left(\psi_{11}\right.$ element) plus this $\left(H_{12}\right.$ element $)$ times this $\left(\psi_{12}\right)$ element. You just get ... Well, if we express those kets, we can't really have a ket times a ket."

Nelson's discussion of this calculation in Dirac notation contains many errors, including inappropriately trying to multiply a row matrix by a square matrix on the left, but we argue that it was the symbolic features of the kets that alerted the student to a potential problem with his calculation. The asymmetric structure of the kets strongly supported Nelson in identifying an error in his calculation (noticing a "ket times a ket" versus a "bra times a ket"). Neither the symmetric parentheses of the "matrices" nor the spatial coordination patterns of matrix multiplication alerted this student to an error.

Next the interviewer asked the student to compute the expectation value using wave-function language. The student confessed that he did not remember how to calculate expectation value using that notation. The interviewer then told him that there are integrals involved, which was familiar to the student. The student integrated $\psi$ times $\psi^{*}$ and recognized that as a probability. He then multiplied that by the Hamiltonian [Figs. 5(b) and 5(c)]. "The integral of $\psi \psi^{*}$ is a probability. So I think we also need it, our expression for $H$ in here somewhere as a function. Integrating with respect to $l$ ?"

He then went back to change the integrand to $\rho$ times $\rho^{*}$ (his symbols for the energy eigenstates) rather than the superposition state $\psi$, but hesitated. "But then this is going back to the summation but that's not what we're going for."

The student changed the integrand again to include the superposition states, and changed the ordering of the elements so that the Hamiltonian was the middle [Fig. 5(d)]. The interviewer then asked what inspired the student to write the last expression, and the student discussed an overview of his entire reasoning process.

"Oh, well [initially] I was thinking of it with just the single components, but then I realized that was only going to give us one part of it because I was thinking about it in terms of this summation with the probability of the first one times that eigenvalue. Then I remembered how we defined probability density with $\psi \psi^{*}$, and then I just kinda remembered just writing this [the correct integrand] down at some point before where, when you just had them all in the functions, you didn't really care about the bra ket stuff because you were just multiplying them together. And so I figured you could just multiply them together and then you'd want to integrate along $x$ from zero to $L$."

According to the student's explanation, the student was at first using the weighted average idea to guide him, but then eventually changed to thinking about the Dirac bracket. We interpret his statement of "you didn't really care about the bra ket stuff" as referring to turning the elements of the Dirac bracket into functions without the
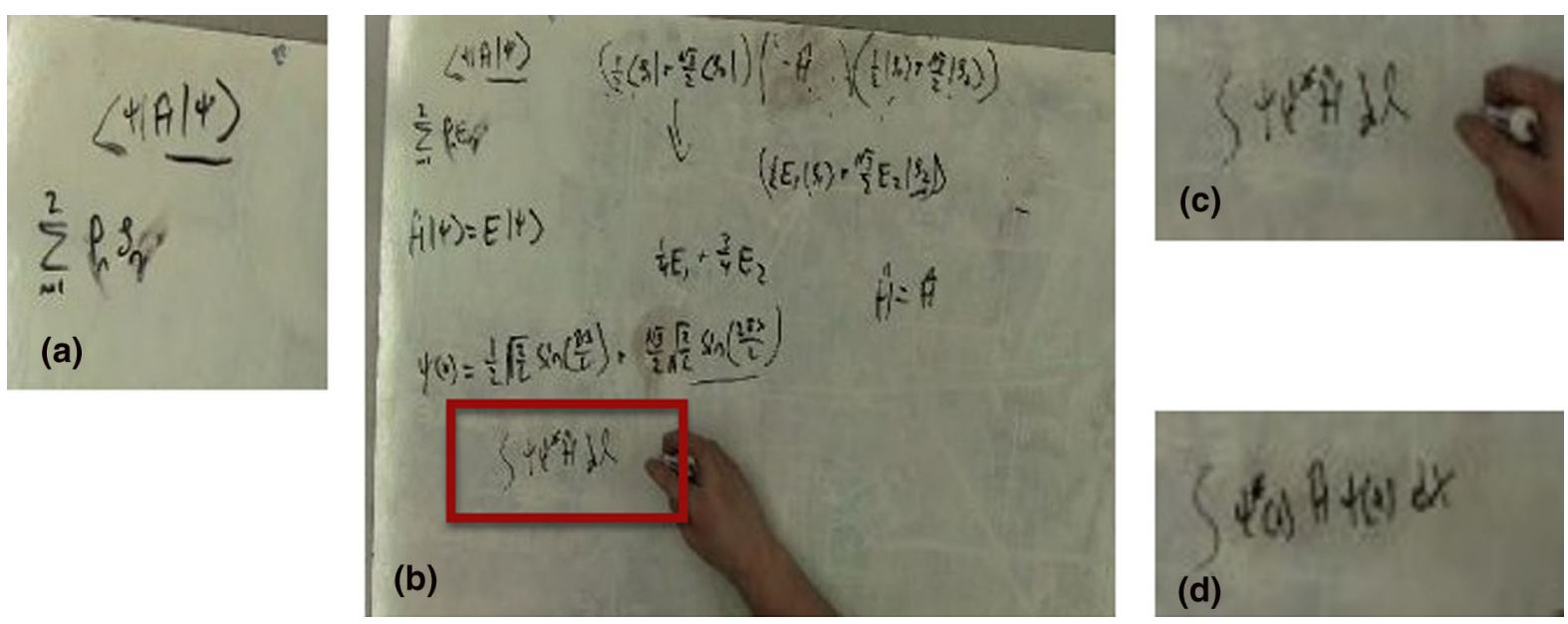

FIG. 5. (a) Beginning of discussion when student describes calculating the expectation value as a weighted average. (b) Full board when the student makes an initial attempt at writing the integral for calculating the energy expectation value (boxed in red). (c) Detail of initial expectation value integral with incorrect ordering of terms in integrand. (d) Detail of the student's second attempt at writing down the integral, after attending to the Dirac bracket for the expectation value. 
symbolic structure of the Dirac notation (the vertical line and the angle bracket) and then multiplying the functions together. Using the bracket as a template led the student to put the integrand elements in the correct order, whereas the weighted average template led to his initial incorrect ordering of integrand elements.

\section{DISCUSSION}

The structural features of the notations can provide an account of the observations noted in Sec. III. The lower error rate and greater progress with Dirac notation, and its value as a template, may be understood by examining the cognitive role of the four structural aspects of these representations (individuation, degree of externalization, compactness, and symbolic support). The high degree of individuation facilitates the identification and manipulation of the two important quantum features (probability amplitudes and basis states). The limited level of externalization of Dirac notation allows the student to perform some calculations without needing to remember the specific details of the quantum state. The high level of compactness of the representation allows for rapid algebraic manipulation of mathematical entities and easier chunking of entities. The high level of symbolic support for computation provides external signals to students about what kinds of manipulations are sensible to do.

Conversely, low levels of symbolic support for computation are consistent with errors in the order of elements. When writing the integrand for an expectation value calculation in wave-function notation, and when writing matrices for an expectation value calculation in matrix notation, students must rely on memory and experience to correctly order the elements; the notation provides little help in this regard.

This work has several implications for instruction. Although some instructors may argue that Dirac notation is sufficiently abstract as to be inappropriate for undergraduate instruction, these interviews provide evidence of Dirac notation productively supporting undergraduate student reasoning about a quantum system. We did see some evidence that these students believed kets to be equivalent to wave functions (states written as functions in a position basis). However, we argue that this subtle conceptual misunderstanding is eclipsed by the computational benefits of Dirac notation, and propose that perhaps equating the ket with the position-basis wave-function eigenstate is a productive intermediate state of conceptual understanding.

The structural aspects of notational systems is a theoretical framework for understanding student performance with external representations, and need not be discussed explicitly with students as part of instruction. However, instructors should discuss with their students the degree of externalization of a notation system, particularly what information is not externalized (e.g., how states vary in space for kets, the basis of a matrix, etc.) in order to facilitate student proficiency with and across notational systems.

This research also suggests that some student difficulties with quantum mechanics may in fact be difficulties with the notational system rather than more fundamental conceptual difficulties (like not understanding the conceptual meaning of expectation value). For example, student Nelson clearly understood the concept of expectation value as weighted average, but had difficulty formulating the computation in either Dirac or wave-function notation. Instructors and researchers should be sensitive to this possibility when attempting to diagnose student difficulties. Another implication is that proficiency in one notation does not extend to proficiency others. Students need practice in multiple notational systems for maximal proficiency.

\section{CONCLUSION}

We have examined three notational systems in quantum mechanics: Dirac notation, algebraic wave-function notation, and matrix notation. An analysis of how the notations represent basis states and their associated complex probability amplitudes resulted in the identification of four features: individuation, degree of externalization, compactness, and symbolic support for computational rules. Problem-solving interviews with advanced undergraduate physics students provided insight into their understanding and coordination of these different representations of quantum systems.

The four features can help explain students' work with the notations. Students readily used Dirac notation, and used a linear combination of kets as a template for a superposition state. We observed students translating between notational systems, using an expression in one notation to guide the development of the analogous expression in another notation. This was typically a productive strategy, but may have been too literal in cases where students failed to appreciate subtleties in the notations, such as equating kets with wave functions in the position basis. The characteristics of individuation, externalization, compactness, and symbolic support for computation vary across Dirac, wave-function, and matrix notations. Dirac notation is highly individuated, can be highly compact, and the symbols support computation, but externalization is limited. Wave-function notation has a high degree of externalization, low individuation and symbolic support, and often limited compactness. Finally, matrix notation has high individuation and can be compact, but has low externalization and limited symbolic support. This work has several implications for instruction, including support for the value and appropriateness of Dirac notation for undergraduates, the importance of having students explicitly explore the varying degrees of externalization in different notation systems, and the need for practice using and coordinating multiple notational systems. 


\section{ACKNOWLEDGMENTS}

We would like to thank the students who volunteered to be interviewed as part of this study, and Corinne Manogue,
Tevian Dray, and Mary Bridget Kustusch for their helpful discussions. This work was supported by NSF Grant No. DUE 1023120.
[1] A. V. Heuvelen, Learning to think like a physicist: A review of research-based instructional strategies, Am. J. Phys. 59, 891 (1991).

[2] P. B. Kohl and N. D. Finkelstein, Patterns of multiple representation use by experts and novices during physics problem solving, Phys. Rev. ST Phys. Educ. Res. 4, 010111 (2008).

[3] D. Rosengrant, A. Van Heuvelen, and E. Etkina, Do students use and understand free-body diagrams?, Phys. Rev. ST Phys. Educ. Res. 5, 010108 (2009).

[4] T. Fredlund, J. Airey, and C. Linder, Exploring the role of physics representations: an illustrative example from students sharing knowledge about refraction, Eur. J. Phys. 33, 657 (2012).

[5] A. F. Heckler, Some consequences of prompting novice students to construct force diagrams, Int. J. Sci. Educ. 32, 1829 (2010).

[6] D. E. Meltzer, Relation between students' problem-solving performance and representational format, Am. J. Phys. 73, 463 (2005).

[7] P. B. Kohl and N. D. Finkelstein, Effects of representation on students solving physics problems: A fine-grained characterization, Phys. Rev. ST Phys. Educ. Res. 2, 010106 (2006).

[8] D. F. Styer, Common misconceptions regarding quantum mechanics, Am. J. Phys. 64, 31 (1996).

[9] I. Johnston, K. Crawford, and P. R. Fletcher, Student difficulties in learning quantum mechanics, Int. J. Sci. Educ. 20, 427 (1998).

[10] G. Ireson, The quantum understanding of pre-university physics students, Phys. Educ. 35, 15 (2000).

[11] C. Singh, Student understanding of quantum mechanics, Am. J. Phys. 69, 885 (2001).

[12] M. C. Wittmann, R. N. Steinberg, and E. F. Redish, Investigating student understanding of quantum physics: Spontaneous models of conductivity, Am. J. Phys. 70, 218 (2002).

[13] S. B. McKagan, K. K. Perkins, and C. E. Wieman, Deeper look at student learning of quantum mechanics: The case of tunneling, Phys. Rev. ST Phys. Educ. Res. 4, 020103 (2008).

[14] E. Cataloglu and R. W. Robinett, Testing the development of student conceptual and visualization understanding in quantum mechanics through the undergraduate career, Am. J. Phys. 70, 238 (2002).

[15] G. Zhu and C. Singh, Surveying students' understanding of quantum mechanics in one spatial dimension, Am. J. Phys. 80, 252 (2012).
[16] G. Zhu and C. Singh, Improving students' understanding of quantum measurement. I. Investigation of difficulties, Phys. Rev. ST Phys. Educ. Res. 8, 010117 (2012).

[17] G. Zhu and C. Singh, Improving student understanding of addition of angular momentum in quantum mechanics, Phys. Rev. ST Phys. Educ. Res. 9, 010101 (2013).

[18] J. Clement, Students' preconceptions in introductory mechanics, Am. J. Phys. 50, 66 (1982).

[19] D. Hammer, Student resources for learning introductory physics, Am. J. Phys. 68, S52 (2000).

[20] A. A. DiSessa, Towards an epistemology of physics, Cognit. Instr. 10, 105 (1993).

[21] G. J. Posner, K. A. Strike, P. W. Hewson, and W. A. Gertzog, Accommodation of a scientific conception: Toward a theory of conceptual change, Sci. Educ. 66, 211 (1982).

[22] D. Kirsh, in Cognition Beyond the Brain, edited by S. J. Cowley and F. Valle-Tourangeau (Springer, London, 2013) pp. 171-194.

[23] M. Cole, Cultural Psychology: A Once and Future Discipline (Harvard University Press, Cambridge, MA, 1998).

[24] E. Hutchins, Cognition in the Wild (MIT Press, Cambridge, MA, 1995).

[25] E. Hutchins, Material anchors for conceptual blends, J. Pragmatics 37, 1555 (2005).

[26] E. Gire and E. Price, Arrows as anchors: An analysis of the material features of electric field vector arrows, Phys. Rev. ST Phys. Educ. Res. 10, 020112 (2014).

[27] C. A. Manogue, P. J. Siemens, J. Tate, K. Browne, M. L. Niess, and A. J. Wolfer, Paradigms in Physics: A new upper-division curriculum, Am. J. Phys. 69, 978 (2001).

[28] C. A. Manogue and K. S. Krane, Paradigms in physics: Restructuring the upper level, Phys. Today 56, No. 9, 53 (2003).

[29] K. A. Ericsson and H. A. Simon, Verbal reports as data, Psychol. Rev. 87, 215 (1980).

[30] D. McIntyre, C. A. Manogue, and J. Tate, Quantum Mechanics: A Paradigms Approach 1st ed. (AddisonWesley, Boston, MA, 2012); D. J. Griffiths, Introduction to Quantum Mechanics, 2nd ed. (Prentice Hall, Upper Saddle River, NJ, 2005).

[31] Hutchins uses the term individuation to describe the idea that one material element is distinguishable from others; in our use, conceptual individuation is important as well. A ket is individuated because we do not recombine the symbols into other expressions, and this is tied to the meaning given to a ket as a single conceptual item. 